\title{
MATHEMATICAL MODELING OF ANOMALOUS DIFFUSION IN POROUS MEDIA
}

\author{
SERGei Fomin, Vladimir Chugunov and Toshiyuki HashidA
}

\begin{abstract}
Analysis of diffusion in a complex environment shows that the conventional diffusion equation based on Fick's law fails to model the anomalous character of the diffusive mass transport observed in the field and laboratory experiments. New mathematical models of diffusive transport, different from Fick's law, were proposed and validated in literature. In the present paper the examples of the equations that can be used for describing the anomalous mass transport are presented and some important properties of these equations are discussed. Two regimes of anomalous diffusion are identified. One regime, which is called sub-diffusion, is characterized by the slower propagation of the concentration front, so that the squared distance of the front passage requires longer time than in the case of the classical Fickian diffusion. The second regime (called super-diffusion) is characterized by the higher diffusion rate, so that the particles will pass the specified distance faster than in the case of classical Fickian diffusion. Both regimes can be modeled by non-local diffusion equation with temporal and spatial fractional derivatives. It is shown that equation with spatially variable diffusivity proposed by O'Shaughnessy and Procaccia (1985), which provides a relatively good model of diffusion on a regular fractal, is less applicable for describing the effects of sub and super diffusion that may take place in a fractured porous medium or any other complex medium.
\end{abstract}

Mathematics subject classification (2010): 35R11, 35Q35, 76S05.

Keywords and phrases: Anomalous diffusion, porous medium, fractional derivative, Laplace transform.

\section{REFERENCES}

[1] M. Abramowitz, I. Stegun, Handbook of mathematical functions with formulas, graphs, and mathematical tables, John Wiley \& Sons, 1972.

[2] V. Afananasiev, R. Sagdeev, G. Zaslavsky, Chaotic jets with multifractal space-tim random walk, Chaos 1(2), 143-159, 1991.

[3] A. V. Akcenov, V. A. Baikov, V. A. Chugunov, R. K. Gazizov, A. G. Meshrov, Lie group analysis of differential equations, Volume 2. Applications in engineering and physical sciences, (ed. N. H. Ibragimov), pp. 1-545, CRC Press, 1994.

[4] V. E. AR KHINCHEEV, A. V. NOMOEV, About nonlinear drift velocity at random walk by Levy Flight: analytical solution and numerical simulations, Physica A 269, 293-298, 1999.

[5] G. I. Barenblatt, V. M. Entov and V. M. Ryzhik, Theory of Fluid Flows Through Natural Rocks, Kluwer Academic Publishers, 1990.

[6] H. Bateman, And A. ERdelyi, Tables of integral transforms, V. 1, p. 344, 1954.

[7] D. A. Benson, S. W. Wheatcraft, And M. M. Meerschaert, The fractional order governing equation of Levy motion, Water Resources Res., 36(6), 1413-1423, 2000.

[8] D. A. Benson, R. Schumer, M. M. Meerschaert, \& S. W. Wheatcraft, Fractional dispersion, Le'vy motion, and the MADE tracer tests, Transp. Porous Media 42, 211-240, 2001.

[9] D. Campos, J. ForT AND V. MendeZ, Propagation through fractal media: The Sierpinski gasket and the Koch curve, Europhysics letters, 68 (6), 769-775, 2004.

[10] A. Compte, Stochastic foundation of fractional dynamics, Phys. Rev. E 53, 4191-4193, 1996. 
[11] D. Del-Castillo-Negrete, B. A. Carreras, And V. E. LynCh, Front dynamics in reactiondiffusion systems with Levy flights: a fractional diffusion approach, Phys. Rev. Lett., 91, 018302(4), 2003.

[12] O. YU. DINARIYEV, The pressure build-up curve for a fractal cracked porous medium, Linear theory, J. Appl. Math. Mech. 58, 755-758, 1994.

[13] Do Hoang Ngoc Anh, K. H. Hoffmann, S. SeEger and S. TARAFdar, Diffusion in disordered fractals, Europhysics letters, 70 (1), 109-115, 2005.

[14] C. Essex, M. Davison, C. Schulsky, A. Franz And K. Hoffmann, The differential equation describing random walks on the Koch curve, J. of Physics A: Math. Gen. 34, 8397-8406, 2001.

[15] S. Fomin, V. ChugunOv AND T. HASHIDA, The effect of non-Fickian diffusion into surrounding rocks on contaminant transport in fractured porous aquifer, Proceedings of Royal Society A 461, 2923-2939, 2005.

[16] S. Fomin, V. Chugunov and T. Hashida, Application of Fractional Differential Equations for Modeling the Anomalous Diffusion of Contaminant from Fracture into Porous Rock Matrix with Bordering Alteration Zone, Transport in Porous Media, 81, 187-205, 2010.

[17] S. A. Fomin, V. A. CHugunov AND T. HASHIDA, Non-Fickian mass transport in fractured porous media, Advances in Water Resources, 2010 (in press, available online, doi:10.1016/j.advwatres.2010.11.002)

[18] M. GIONA AND H. E. Roman, Fractional diffusion equation on fractals: one-dimensional case and asymptotic behavior, J. Phys. A: Math. Gen. 25, 2093-2105, 1992.

[19] S. Havlin and D. Ben-Avraham, Diffusion in disordered media, Advances in Physics 51(1), 187-292, 2002.

[20] F. HUANG AND F. LiU, The time fractional diffusion equation and the advection-dispersion equation, ANZIAM J. 46, 317-330, 2005.

[21] N. H. Ibragimov, Transformation Groups Applied in Mathematical Physics, D. Reidel Publ., Dordrecht, 1985.

[22] J. Klafter, A. Blumen, G. Zumofen And M. F. Shlesinger, Levy walk approach to anomalous diffusion, Physica A 168, 637-645, 1990.

[23] M. M. Meerschaert, D. A. Benson, H.-P. Scheffler and P. Becker-Kern, Governing equations and solutions of anomalous random walk limits, Phys. Rev. E 66, 060102 (R), 2002a.

[24] M. M. Meerschaert, D. A. Benson, And H.-P. SchefFler, Stochastic solution of space-time fractional diffusion equations, Phys. Rev. E, 65, 041103(4), 2002b.

[25] R. METZler, AND J. KLAFTER, The random walk's guide to anomalous diffusion: a fractional dynamics approach, Physics Reports 339, 1-77, 2000.

[26] R. R. Nigmatullin, To the theoretical explanation of the "universal response", Phys. Stat. Sol.(b) 123, 739-745, 1984.

[27] R. R. Nigmatullin, On the theory of relaxation for systems with "remnant memory", Phys. Stat. Sol. (b) 124, 389-393, 1984.

[28] R. R. Nigmatullin, The realization of the generalized transfer equation in a medium with fractal geometry, Phys. Stat. Sol. (b), 133, 425-430, 1986.

[29] B. O’Shaughnessy, And I. Procaccia, Diffusion on fractals, Phys. Rev. A 32, 3073-3083, 1985.

[30] S. REDNER, Supperdiffusion in random velocity fields, Physica A, 168, 551-560, 1990.

[31] S. G. SAMko, A. A. KIlbas, \& O. I. MARICHEV, Fractional Integrals and Derivatives: Theory and Applications, Gordon and Breach, London, 1993.

[32] C. Schulzky, C. Essex, M. Davison, A. Franz and K. H. Hoffmann, The similarity group and anomalous diffusion equations, J. Phys. A: Math. Gen. 33, 5501-5511, 2000.

[33] R. T. Sibatov, AND V. V. UChaikin, Fractional differential approach to dispersive transport in semiconductors, Uspekhi Fizicheskih Nauk 179(10), 1079-1104, 2009.

[34] V. V. Uchaikin, The Fractional Derivatives Method, Artishok Press, Ul'yanovsk, 512 p., 2008.

[35] G. M. Zaslavsky, Chaos, fractional kinetics, and anomalous transport, Physics Reports 371, 461580, 2002. 